\title{
Evaluation of the Missouri WIC (Special Supplemental Nutrition Program for Women, Infants, and Children) breast-feeding peer counselling programme
}

\author{
Shumei Yun*t, Qian Liu, Kathy Mertzlufft, Catherine Kruse, Maggie White, Phyllis Fuller \\ and Bao-Ping Zhu \\ Division of Community and Public Health, Missouri Department of Health and Senior Services, Jefferson City, \\ MO, USA
}

Submitted 6 March 2008: Accepted 22 May 2009: First published online 17 July 2009

\begin{abstract}
Objective: To evaluate the effectiveness of the peer counselling (PC) programme on breast-feeding initiation among participants in the Special Supplemental Nutrition Program for Women, Infants, and Children (WIC) in Missouri, and to identify factors that facilitate breast-feeding initiation.

Design: We used the data from the 2006 Missouri Pregnancy Nutrition Surveillance System, Missouri Live Birth Records and the Missouri WIC programme to compare breast-feeding initiation rates between PC and non-PC agencies. We used multilevel logistic regression, with individual participants being nested within agencies, to control for individual- and agency-level characteristics.

Results: The breast-feeding initiation rate in PC agencies was significantly higher than in non-PC agencies among prenatal participants, but the difference was not significant among postpartum participants. After controlling for maternal sociodemographic characteristics, compared with prenatal cases in non-PC agencies, prenatal cases in PC agencies were more likely to initiate breast-feeding ( $\mathrm{OR}=$ $1 \cdot 21 ; 95 \%$ CI $1 \cdot 03,1 \cdot 43$ ), whereas postpartum cases were less likely to initiate breast-feeding. Among prenatal participants in PC agencies, longer duration of prenatal WIC enrolment was associated with a higher rate of breast-feeding initiation. After adjusting for maternal sociodemographic characteristics and other agency-level characteristics, participants of PC agencies with an international board-certified lactation consultant were more likely to initiate breast-feeding than participants of PC agencies without such a consultant $(\mathrm{OR}=1 \cdot 21 ; 95 \% \mathrm{CI}$ $1 \cdot 01,1 \cdot 45)$.

Conclusions: Prenatal participation in the WIC breast-feeding PC programme (especially participation early during pregnancy) was associated with an increased rate of breast-feeding initiation in Missouri.
\end{abstract}

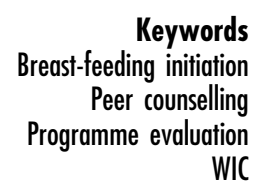

Breast-feeding has been shown to benefit both the baby and the mother in many studies ${ }^{(1-5)}$. A number of randomized controlled trials have shown that peer counselling (PC) can help to increase breast-feeding initiation and duration ${ }^{(6-8)}$. In 1992, the Missouri Special Supplemental Nutrition Program for Women, Infants, and Children (WIC) began dedicating a portion of its funds to local agencies to begin offering PC services. The purpose of this project was to enhance the breast-feeding promotion and support activities already provided by WIC.

In 2004, the United States Department of Agriculture (USDA) through the Food and Nutrition Services (FNS) implemented the 'Loving Support' model for successful PC.

$\uparrow$ Correspondence address: PO Box 47835, Olympia, WA 98504, USA.
The purpose of this model was to aid state agencies in instituting best practice and training to build and sustain successful PC programmes. In fiscal year 2004, \$US 14.9 million were appropriated nationwide for this additional WIC breast-feeding support and \$US 14.8 million were appropriated nationwide in both fiscal year 2005 and fiscal year $2006^{(9)}$. The state of Missouri has been using this additional funding to enhance its existing programme.

In 2006, forty-four WIC agencies, out of a total of 118, participated in the PC programme in Missouri. Each local agency assigns someone to fill the role of PC coordinator. The PC coordinator may be a dietitian, a nurse or an international board-certified lactation consultant (IBCLC), and is responsible for supervising and mentoring the peer counsellors and facilitating the programme at the local 
level. The PC coordinator and peer counsellors are required to attend state-sponsored training that utilizes the 'Loving Support' curriculum developed by the USDA and FNS.

Since the start of the PC programme in Missouri, no formal study has been conducted to evaluate its effectiveness on breast-feeding initiation and duration among Missouri WIC participants. Evaluations of several PC programmes in other states have shown that these programmes are effective in improving breast-feeding initiation and duration among WIC participants ${ }^{(10-15)}$. However, these studies typically included only a few WIC agencies and only a few hundred WIC participants. Only one evaluation study, conducted in Mississippi, included Pediatric Nutrition Surveillance System (PedNSS) data from children in the state ${ }^{(15)}$. Since the demographics of WIC participants and the operation of PC programmes differ in each state, whether the Mississippi experience could be generalized to other states is unknown.

The present study was conducted to evaluate the effectiveness of the Missouri WIC breast-feeding PC program on breast-feeding initiation, and to identify factors in the PC agencies that facilitated breast-feeding initiation among Missouri WIC participants.

\section{Methods}

The 2006 Missouri Pregnancy Nutrition Surveillance System (PNSS) data were used in the present study. PNSS is an ongoing programme-based surveillance system that monitors maternal health and behavioural indicators associated with birth outcomes among low-income pregnant women participating in the WIC. All 118 WIC agencies in Missouri collected data on demographic, health and behavioural indicators (including breast-feeding initiation) from women during prenatal and postpartum clinic visits and contributed these data to PNSS. All of the 2006 Missouri WIC participants who gave birth in 2006 were included in the present study. These included 29390 WIC participants; 15788 were in PC agencies (12551 prenatal and 3237 postpartum cases) and 13602 were in non-PC agencies (10 791 prenatal and 2811 postpartum cases). Prenatal cases are those who enrolled in WIC before delivery, whereas postpartum cases are those who enrolled after delivery.

To obtain information on newborn health status, delivery and labour complications, and the method of delivery, the PNSS data were linked to the 2006 Missouri birth certificate data using the mother's social security number or the mother's full name. Data on the characteristics of WIC agencies were collected during day-to-day programme operations and maintained by the programme epidemiologist. Missouri birth certificate data and PNSS data were cleaned and managed by the surveillance programme staff. Missouri is recognized nationally for its high-quality surveillance data.
The effect of PC on breast-feeding initiation was examined by comparing the breast-feeding initiation rates between PC agencies and non-PC agencies. Multilevel logistic regression procedures, with individual participants (level 1) being nested within agencies (level 2), were used when comparing PC and non-PC agencies. The interactions between PC and maternal sociodemographic characteristics and method of delivery were tested and found to be not significant. The analysis first adjusted for maternal age, race, education and family income. Maternal and infant characteristics, including maternal employment status, maternal smoking and drinking during pregnancy, pre-pregnancy weight, parity, preterm delivery, method of delivery and prenatal care status, were further adjusted. The GLLAMM procedure in the STATA statistical software package version SE $8 \cdot 2$ (StataCorp, College Station, TX, USA) was used for the multilevel analysis.

Since the PC programme is unlikely to have an impact on breast-feeding initiation among postpartum cases, we kept the postpartum cases in both PC and non-PC agencies in the analysis as comparison groups. If $\mathrm{PC}$ helped in improving breast-feeding initiation, we would find the breast-feeding initiation rate to be higher in PC agencies than in non-PC agencies among prenatal cases, but not among postpartum cases. The four combinations of the two variables (prenatal enrolment status and agency PC status) were constructed and included in the multilevel model: prenatal cases in PC agencies, prenatal cases in non-PC agencies, postpartum cases in PC agencies and postpartum cases in non-PC agencies. To identify PC agency-level characteristics that are associated with breast-feeding initiation, multilevel analyses were performed with breast-feeding initiation as the individual-level outcome variable and selected agency-level characteristics as exposure variables. Potential confounding factors at individual and agency levels were controlled in the analysis.

The Institutional Review Board at the Missouri Department of Health and Senior Services reviewed and approved the study protocol.

\section{Results}

\section{Characteristics of Missouri WIC agencies}

Compared with non-PC agencies, PC agencies were more likely to be located in east, north-west and south-west regions, and less likely to be in north-east and south-east regions. Among the forty-four PC agencies, eighteen $(40.9 \%)$ had less than 4 years of peer-counselling experience, sixteen $(36 \cdot 4 \%)$ had 5-8 years of experience and ten (22.7\%) had 9-14 years of experience. In addition, fifteen agencies $(34 \cdot 1 \%)$ had an IBCLC, seven $(15 \cdot 9 \%)$ had peer counsellors who also had other positions in the agencies, and six (13.6\%) had PC coordinators who were a Missouri Breastfeeding PC Task Force member (Table 1). 
Table 1 Characteristics of Missouri WIC agencies by PC status, 2006

\begin{tabular}{|c|c|c|c|c|c|c|}
\hline & \multicolumn{2}{|c|}{$\begin{array}{c}\text { Non-PC agency } \\
(n 74)\end{array}$} & \multicolumn{2}{|c|}{$\begin{array}{l}\text { PC agency } \\
\quad(n \text { 44) }\end{array}$} & \multicolumn{2}{|c|}{$\begin{array}{l}\text { Total } \\
(n 118)\end{array}$} \\
\hline & $n$ & $\%$ & $n$ & $\%$ & $n$ & $\%$ \\
\hline \multicolumn{7}{|l|}{ Region } \\
\hline North-west/Cameron & 7 & $9 \cdot 5$ & 5 & $11 \cdot 4$ & 12 & $10 \cdot 2$ \\
\hline North-east & 19 & $25 \cdot 7$ & 2 & $4 \cdot 5$ & 21 & $17 \cdot 8$ \\
\hline Central & 13 & $17 \cdot 6$ & 8 & $18 \cdot 2$ & 21 & $17 \cdot 8$ \\
\hline South-east & 18 & $24 \cdot 3$ & 7 & $15 \cdot 9$ & 25 & $21 \cdot 2$ \\
\hline South-west & 11 & $14 \cdot 9$ & 10 & $22 \cdot 7$ & 21 & $17 \cdot 8$ \\
\hline North-west/Metro & 4 & $5 \cdot 4$ & 6 & $13 \cdot 6$ & 10 & $8 \cdot 5$ \\
\hline East & 2 & $2 \cdot 7$ & 6 & $13 \cdot 6$ & 8 & $6 \cdot 8$ \\
\hline \multicolumn{7}{|c|}{ Years of experience in $\mathrm{PC}$} \\
\hline $1-4$ & - & - & 18 & $40 \cdot 9$ & 18 & $40 \cdot 9$ \\
\hline $5-9$ & - & - & 16 & $36 \cdot 4$ & 16 & $36 \cdot 4$ \\
\hline $9-14$ & - & - & 10 & $22 \cdot 7$ & 10 & $22 \cdot 7$ \\
\hline \multicolumn{7}{|l|}{ Agency has IBCLC } \\
\hline Yes & - & - & 15 & $34 \cdot 1$ & 15 & $34 \cdot 1$ \\
\hline No & - & - & 29 & $65 \cdot 9$ & 29 & $65 \cdot 9$ \\
\hline \multicolumn{7}{|c|}{ Peer counsellor has other positions in WIC agency } \\
\hline Yes & - & - & 7 & $15 \cdot 9$ & 7 & $15 \cdot 9$ \\
\hline No & - & - & 37 & $84 \cdot 1$ & 37 & $84 \cdot 1$ \\
\hline \multicolumn{7}{|c|}{ Breast-feeding coordinator is Breastfeeding PC Task Force member } \\
\hline Yes & - & - & 6 & $13 \cdot 6$ & 6 & $13 \cdot 6$ \\
\hline No & - & - & 38 & $86 \cdot 4$ & 38 & $86 \cdot 4$ \\
\hline
\end{tabular}

WIC, Special Supplemental Nutrition Program for Women, Infants, and Children; PC, peer counselling; IBCLC, international board-certified lactation consultant.

\section{Characteristics of Missouri WIC participants}

The majority of WIC participants in PC agencies and nonPC agencies were non-Hispanic white. Compared with participants of non-PC agencies, participants in PC agencies were more likely to be non-Hispanic black, to have fewer years of education, to have a lower monthly family income and to enrol in WIC less than 6 months before delivery, but were less likely to smoke or drink during pregnancy. Compared with prenatal cases, for both non-PC and $\mathrm{PC}$ agencies, postpartum cases were more likely to be non-Hispanic black, to be older and to have more years of education. On the other hand, they were less likely to smoke or drink during pregnancy, to be obese before pregnancy and to have late or no prenatal care. Also, infants of postpartum cases were more likely to have a low $(<2500 \mathrm{~g})$ birth weight and less likely to have a high ( $\geq 4000 \mathrm{~g}$ ) birth weight and to be first-born (Table 2).

\section{Breast-feeding initiation rates by agency-and individual-level characteristics}

Breast-feeding initiation rates varied by WIC region: the rate was highest in the south-west region (58.0\%) and lowest in the south-east region (39.9\%). Breast-feeding initiation rates varied by maternal social demographic characteristics: among the racial groups, the rate was highest among Hispanics and lowest among non-Hispanic blacks. The rate was higher among older mothers and increased with higher maternal education level and family income. Also, the rate was higher among employed mothers than unemployed mothers (Table 3).

Mothers who smoked during pregnancy were less likely to initiate breast-feeding compared with those who did not. On the other hand, mothers who drank alcohol during pregnancy were more likely to initiate breastfeeding compared with those who did not. Mothers who were underweight during the pre-pregnancy period were less likely to initiate breast-feeding than mothers who had normal weight or were overweight. Mothers who delivered infants of normal birth weight had higher breastfeeding initiation rate than those who delivered infants of low or high birth weight; similarly, mothers who delivered full-term infants were more likely to initiate breast-feeding than those who delivered preterm infants. Mothers who delivered through Caesarean section had slightly lower initiation rates. The initiation rate was higher among firsttime mothers and mothers who had early prenatal care. The above relationships were observed in both $\mathrm{PC}$ and non-PC agencies, and among prenatal and postpartum cases, with only a few exceptions (Table 3).

\section{Comparisons of breast-feeding initiation between agencies with and without peer counselling}

The difference in breast-feeding initiation rates between PC and non-PC agencies varied between prenatal and postpartum cases. Among prenatal cases, the breast-feeding initiation rate in $\mathrm{PC}$ agencies was $2 \cdot 3$ percentage points higher than non-PC agencies, which was statistically significant; however, among postpartum cases, the rate in PC agencies was 1.4 percentage points lower than that in nonPC agencies and the difference was not significant. The multilevel analysis showed that, compared with prenatal cases in non-PC agencies, prenatal cases in $\mathrm{PC}$ agencies were more likely to initiate breast-feeding (OR $=1 \cdot 18 ; 95 \%$ CI 1·00, 1.40), whereas postnatal cases in PC agencies 
Table 2 Characteristics of WIC participants by agency PC status and participant enrolment status, Missouri, 2006

\begin{tabular}{|c|c|c|c|c|c|}
\hline & \multicolumn{2}{|c|}{ Non-PC agency } & \multicolumn{2}{|c|}{ PC agency } & \multirow[b]{2}{*}{ Total (\%) } \\
\hline & $\begin{array}{c}\text { Prenatal cases } \\
(\%)\end{array}$ & $\begin{array}{c}\text { Postnatal cases } \\
(\%)\end{array}$ & $\begin{array}{c}\text { Prenatal cases } \\
(\%)\end{array}$ & $\begin{array}{c}\text { Postnatal cases } \\
(\%)\end{array}$ & \\
\hline Number of participants & n 10791 & $n 2811$ & n 12551 & $n 3237$ & $n 29881^{*}$ \\
\hline \multicolumn{6}{|l|}{ Maternal demographics } \\
\hline \multicolumn{6}{|l|}{ Maternal race/ethnicity } \\
\hline White, non-Hispanic & $76 \cdot 3$ & $66 \cdot 6$ & $62 \cdot 5$ & $57 \cdot 7$ & $67 \cdot 4$ \\
\hline Black, non-Hispanic & $13 \cdot 7$ & $24 \cdot 5$ & $26 \cdot 1$ & $32 \cdot 6$ & $22 \cdot 1$ \\
\hline Hispanic & $7 \cdot 9$ & $6 \cdot 6$ & $8 \cdot 7$ & $6 \cdot 6$ & $8 \cdot 1$ \\
\hline American Indian/Alaskan Native & $1 \cdot 1$ & $0 \cdot 8$ & $1 \cdot 3$ & $1 \cdot 1$ & $1 \cdot 1$ \\
\hline Asian/Pacific Islander & 0.9 & $1 \cdot 2$ & $1 \cdot 1$ & $1 \cdot 4$ & $1 \cdot 1$ \\
\hline All other & $0 \cdot 1$ & $0 \cdot 3$ & $0 \cdot 3$ & $0 \cdot 6$ & $0 \cdot 2$ \\
\hline \multicolumn{6}{|l|}{ Maternal age } \\
\hline$<20$ years & 24.9 & $13 \cdot 6$ & $24 \cdot 4$ & $13 \cdot 1$ & $22 \cdot 3$ \\
\hline $20-29$ years & $62 \cdot 1$ & $67 \cdot 2$ & $61 \cdot 3$ & $68 \cdot 3$ & $63 \cdot 0$ \\
\hline 30-39 years & $12 \cdot 3$ & $18 \cdot 4$ & $13 \cdot 1$ & $17 \cdot 4$ & $13 \cdot 8$ \\
\hline$\geq 40$ years & $0 \cdot 7$ & $0 \cdot 7$ & $0 \cdot 9$ & $1 \cdot 2$ & $0 \cdot 9$ \\
\hline \multicolumn{6}{|l|}{ Maternal education } \\
\hline$<12$ years & $34 \cdot 7$ & $27 \cdot 6$ & $37 \cdot 4$ & $30 \cdot 5$ & $34 \cdot 5$ \\
\hline 12 years & $46 \cdot 1$ & $44 \cdot 5$ & $42 \cdot 9$ & $42 \cdot 1$ & $44 \cdot 2$ \\
\hline$>12$ years & $19 \cdot 2$ & $27 \cdot 9$ & $19 \cdot 7$ & $27 \cdot 4$ & $21 \cdot 3$ \\
\hline \multicolumn{6}{|l|}{ Monthly household income } \\
\hline \$US 0-500 & $27 \cdot 7$ & $33 \cdot 2$ & $30 \cdot 5$ & $38 \cdot 6$ & $30 \cdot 7$ \\
\hline \$US 501-1000 & $18 \cdot 0$ & $15 \cdot 2$ & $19 \cdot 0$ & $14 \cdot 1$ & $17 \cdot 7$ \\
\hline \$US 1001-1500 & $18 \cdot 7$ & $15 \cdot 3$ & $18 \cdot 3$ & $13 \cdot 9$ & $17 \cdot 6$ \\
\hline \$US 1501-2000 & $15 \cdot 3$ & $13 \cdot 2$ & $15 \cdot 0$ & $13 \cdot 3$ & $14 \cdot 8$ \\
\hline >\$US 2000 & $20 \cdot 2$ & $23 \cdot 1$ & $17 \cdot 1$ & $20 \cdot 1$ & $19 \cdot 2$ \\
\hline \multicolumn{6}{|l|}{ Other maternal health indicators } \\
\hline Maternal smoking during pregnancy & $42 \cdot 9$ & $35 \cdot 1$ & $38 \cdot 6$ & $34 \cdot 3$ & $39 \cdot 2$ \\
\hline Maternal drinking during pregnancy & $12 \cdot 1$ & $9 \cdot 9$ & $10 \cdot 7$ & $6 \cdot 4$ & $10 \cdot 6$ \\
\hline \multicolumn{6}{|c|}{ Maternal pre-pregnancy weight status ( $n 27316)$} \\
\hline Underweight & $5 \cdot 8$ & $4 \cdot 9$ & $5 \cdot 7$ & 4.5 & $5 \cdot 5$ \\
\hline Normal weight & $42 \cdot 0$ & $46 \cdot 3$ & $42 \cdot 6$ & $46 \cdot 6$ & $43 \cdot 2$ \\
\hline Overweight & $22 \cdot 9$ & $24 \cdot 0$ & $22 \cdot 8$ & $23 \cdot 0$ & $23 \cdot 0$ \\
\hline Obese & $29 \cdot 3$ & $24 \cdot 8$ & $28 \cdot 9$ & $26 \cdot 0$ & $28 \cdot 3$ \\
\hline \multicolumn{6}{|l|}{ Newborn indicators } \\
\hline \multicolumn{6}{|l|}{ Birth weight } \\
\hline Low birth weight $(<2500 \mathrm{~g})$ & $7 \cdot 0$ & $9 \cdot 7$ & $7 \cdot 7$ & $9 \cdot 9$ & $7 \cdot 9$ \\
\hline Normal birth weight $(2500-3999 \mathrm{~g})$ & $83 \cdot 1$ & $81 \cdot 8$ & $83 \cdot 7$ & $82 \cdot 0$ & $83 \cdot 1$ \\
\hline High birth weight $(\geq 4000 \mathrm{~g})$ & $9 \cdot 8$ & $8 \cdot 5$ & $8 \cdot 7$ & $8 \cdot 2$ & $9 \cdot 0$ \\
\hline Preterm delivery ( $n$ 29 850) & $12 \cdot 1$ & $14 \cdot 2$ & $12 \cdot 2$ & $14 \cdot 4$ & $12 \cdot 6$ \\
\hline \multicolumn{6}{|l|}{ Parity ( $n$ 29867) } \\
\hline 1st birth & $43 \cdot 1$ & $30 \cdot 8$ & $43 \cdot 3$ & $31 \cdot 0$ & $40 \cdot 6$ \\
\hline 2nd-4th birth & $53 \cdot 1$ & $61 \cdot 7$ & $52 \cdot 0$ & $61 \cdot 1$ & $54 \cdot 4$ \\
\hline 5th or more birth & $3 \cdot 8$ & $7 \cdot 5$ & $4 \cdot 7$ & $7 \cdot 9$ & $5 \cdot 0$ \\
\hline \multicolumn{6}{|l|}{ Method of delivery } \\
\hline Caesarean section ( $n$ 27705) & $29 \cdot 4$ & $32 \cdot 5$ & $28 \cdot 2$ & $31 \cdot 3$ & $29 \cdot 3$ \\
\hline \multicolumn{6}{|l|}{ WIC enrolment date ( $n$ 29 355) } \\
\hline$<3$ months before delivery & $19 \cdot 7$ & - & $20 \cdot 1$ & - & $15 \cdot 8$ \\
\hline $3-6$ months before delivery & $30 \cdot 0$ & - & $33 \cdot 5$ & - & $25 \cdot 4$ \\
\hline$>6$ months before delivery & $50 \cdot 2$ & - & $46 \cdot 4$ & - & $38 \cdot 3$ \\
\hline$<1$ month after delivery & - & $63 \cdot 0$ & - & $65 \cdot 5$ & $13 \cdot 2$ \\
\hline$>1$ month after delivery & - & $37 \cdot 0$ & - & $34 \cdot 5$ & $7 \cdot 2$ \\
\hline Late or no prenatal caret & $21 \cdot 0$ & $7 \cdot 6$ & $21 \cdot 9$ & $8 \cdot 8$ & 18.5 \\
\hline
\end{tabular}

WIC, Special Supplemental Nutrition Program for Women, Infants, and Children; PC, peer counselling.

*The total number of WIC participants who gave birth in the year 2006 was 29881 . However, 491 cases had missing values on the variable 'participant enrolment status', resulting in a combined total of 29390 for the prenatal cases and the postnatal cases.

tLate or no prenatal care is the percentage of births that occurred to mothers who reported receiving prenatal care only in the third trimester of their pregnancy, or reported receiving no prenatal care on their child's birth certificate.

$(\mathrm{OR}=0 \cdot 88 ; 95 \% \mathrm{CI} 0 \cdot 73,1 \cdot 05)$ and postnatal cases in non$\mathrm{PC}$ agencies $(\mathrm{OR}=0 \cdot 85 ; 95 \% \mathrm{CI} 0 \cdot 78,0 \cdot 93)$ were less likely to initiate breast-feeding. After controlling for maternal sociodemographic characteristics, prenatal cases in PC agencies (OR $=1 \cdot 21 ; 95 \%$ CI 1·03, 1·43) were more likely to initiate breast-feeding, whereas postnatal cases in PC agencies
$(\mathrm{OR}=0 \cdot 86 ; 95 \% \mathrm{CI} 0 \cdot 72,1 \cdot 02)$ and postnatal cases in non-PC agencies $(\mathrm{OR}=0 \cdot 81 ; 95 \% \mathrm{CI} 0 \cdot 74,0 \cdot 89)$ were less likely to initiate breast-feeding, compared with prenatal cases in nonPC agencies. After further adjusting for maternal smoking, maternal drinking, maternal pre-pregnancy weight status, parity, preterm delivery, method of delivery, prenatal care 
Table 3 Breast-feeding initiation rates in PC and non-PC agencies by selected agency and participant characteristics 


\begin{tabular}{|c|c|c|c|c|c|c|c|c|c|c|}
\hline & \multicolumn{10}{|c|}{ Breast-feeding initiation rate } \\
\hline & \multicolumn{4}{|c|}{ Non-PC agency ( $n$ 13602) } & \multicolumn{4}{|c|}{ PC agency ( $n$ 15788) } & & \\
\hline & \multicolumn{2}{|c|}{ Prenatal cases } & \multicolumn{2}{|c|}{ Postnatal cases } & \multicolumn{2}{|c|}{ Prenatal cases } & \multicolumn{2}{|c|}{ Postnatal cases } & \multicolumn{2}{|c|}{ Total $\left(n 29881^{\star}\right)$} \\
\hline & $\%$ & $95 \% \mathrm{Cl}$ & $\%$ & $95 \% \mathrm{Cl}$ & $\%$ & $95 \% \mathrm{Cl}$ & $\%$ & $95 \% \mathrm{Cl}$ & $\%$ & $95 \% \mathrm{Cl}$ \\
\hline \multicolumn{11}{|c|}{ Maternal pre-pregnancy weight status ( $n 27316$ ) } \\
\hline Underweight & $44 \cdot 6$ & $40 \cdot 5,48 \cdot 7$ & $36 \cdot 7$ & $28 \cdot 4,45 \cdot 1$ & $44 \cdot 6$ & $40 \cdot 8,48 \cdot 4$ & $36 \cdot 1$ & $27 \cdot 9,44 \cdot 3$ & $44 \cdot 0$ & $41 \cdot 5,46 \cdot 5$ \\
\hline Normal weight & $46 \cdot 4$ & $44 \cdot 9,47 \cdot 9$ & $44 \cdot 2$ & $41 \cdot 4,47 \cdot 0$ & $50 \cdot 4$ & $49 \cdot 0,51 \cdot 8$ & $43 \cdot 6$ & $40 \cdot 9,46 \cdot 2$ & $48 \cdot 3$ & $47 \cdot 4,49 \cdot 2$ \\
\hline Overweight & $48 \cdot 3$ & $46 \cdot 3,50 \cdot 4$ & $45 \cdot 5$ & $41 \cdot 6,49 \cdot 4$ & $50 \cdot 3$ & $48 \cdot 4,52 \cdot 2$ & $42 \cdot 2$ & $38 \cdot 5,45 \cdot 9$ & $49 \cdot 1$ & $47 \cdot 8,50 \cdot 3$ \\
\hline Obese & $46 \cdot 3$ & $44 \cdot 4,48 \cdot 1$ & $44 \cdot 4$ & $40 \cdot 6,48 \cdot 3$ & $48 \cdot 3$ & $46 \cdot 5,50 \cdot 0$ & $41 \cdot 7$ & $38 \cdot 2,45 \cdot 1$ & $47 \cdot 1$ & $46 \cdot 0,48 \cdot 2$ \\
\hline \multicolumn{11}{|l|}{ Newborn indicators } \\
\hline \multicolumn{11}{|l|}{ Birth weight } \\
\hline Low birth weight $(<2500 \mathrm{~g})$ & $47 \cdot 6$ & $44 \cdot 0,51 \cdot 1$ & $38 \cdot 0$ & $32 \cdot 2,43 \cdot 7$ & $49 \cdot 2$ & $46 \cdot 0,52 \cdot 3$ & $44 \cdot 2$ & $38 \cdot 8,49 \cdot 7$ & $47 \cdot 7$ & $45 \cdot 6,49 \cdot 7$ \\
\hline Normal birth weight $(2500-3999 \mathrm{~g})$ & $49 \cdot 5$ & $48 \cdot 5,50 \cdot 5$ & $45 \cdot 5$ & $43 \cdot 5,47 \cdot 6$ & $52 \cdot 0$ & $51 \cdot 1,53 \cdot 0$ & $44 \cdot 1$ & $42 \cdot 2,46 \cdot 0$ & $50 \cdot 4$ & $49 \cdot 8,51 \cdot 0$ \\
\hline High birth weight ( $\geq 4000 \mathrm{~g}$ ) & $43 \cdot 4$ & $40 \cdot 4,46 \cdot 4$ & $50 \cdot 4$ & $44 \cdot 1,56 \cdot 8$ & $43 \cdot 7$ & $40 \cdot 7,46 \cdot 6$ & $39 \cdot 8$ & $33 \cdot 9,45 \cdot 7$ & $44 \cdot 3$ & $42 \cdot 4,46 \cdot 2$ \\
\hline \multicolumn{11}{|l|}{ Preterm delivery ( $n$ 29850) } \\
\hline Yes & $39 \cdot 5$ & $36 \cdot 8,42 \cdot 1$ & $38 \cdot 4$ & $33 \cdot 6,43 \cdot 2$ & $43 \cdot 2$ & $40 \cdot 7,45 \cdot 6$ & $34 \cdot 3$ & $30 \cdot 0,38 \cdot 7$ & $41 \cdot 0$ & $39 \cdot 4,42 \cdot 6$ \\
\hline No & $50 \cdot 0$ & $49 \cdot 0,51 \cdot 0$ & $46 \cdot 1$ & $44 \cdot 1,48 \cdot 1$ & $52 \cdot 2$ & $51 \cdot 2,53 \cdot 1$ & $45 \cdot 2$ & $43 \cdot 3,47 \cdot 0$ & $50 \cdot 9$ & $50 \cdot 2,51 \cdot 5$ \\
\hline \multicolumn{11}{|l|}{ Parity (n 29 867) } \\
\hline 1st birth & $51 \cdot 4$ & $50 \cdot 0,52 \cdot 9$ & $54 \cdot 3$ & $51 \cdot 0,57 \cdot 7$ & $55 \cdot 0$ & $53 \cdot 6,56 \cdot 3$ & $54 \cdot 8$ & $51 \cdot 7,57 \cdot 9$ & $54 \cdot 2$ & $53 \cdot 3,55 \cdot 0$ \\
\hline 2nd-4th birth & $46 \cdot 6$ & $45 \cdot 3,47 \cdot 9$ & $42 \cdot 0$ & $39 \cdot 6,44 \cdot 3$ & $48 \cdot 1$ & $46 \cdot 8,49 \cdot 3$ & $39 \cdot 6$ & $37 \cdot 4,41 \cdot 7$ & $46 \cdot 7$ & $45 \cdot 9,47 \cdot 5$ \\
\hline 5th or more birth & $48 \cdot 5$ & $45 \cdot 4,53 \cdot 4$ & $34 \cdot 9$ & $28 \cdot 5,41 \cdot 3$ & $48 \cdot 7$ & $44 \cdot 7,52 \cdot 7$ & $32 \cdot 8$ & $27 \cdot 1,38 \cdot 6$ & $45 \cdot 0$ & $42 \cdot 4,47 \cdot 5$ \\
\hline \multicolumn{11}{|c|}{ Method of delivery - Caesarean section ( $n$ 27 705) } \\
\hline Yes & $48 \cdot 1$ & $46 \cdot 3,49 \cdot 9$ & $47 \cdot 2$ & $43 \cdot 8,50 \cdot 7$ & $51 \cdot 3$ & $49 \cdot 7,53 \cdot 0$ & $47 \cdot 7$ & $44 \cdot 4,51 \cdot 0$ & $50 \cdot 0$ & $48 \cdot 9,51 \cdot 1$ \\
\hline No & $50 \cdot 1$ & $49 \cdot 0,51 \cdot 3$ & $43 \cdot 9$ & $41 \cdot 6,46 \cdot 3$ & $52 \cdot 0$ & $50 \cdot 9,53 \cdot 0$ & $42 \cdot 1$ & $39 \cdot 9,44 \cdot 3$ & $50 \cdot 4$ & $49 \cdot 7,51 \cdot 1$ \\
\hline \multicolumn{11}{|l|}{ Late or no prenatal care } \\
\hline Yes & $46 \cdot 5$ & $44 \cdot 5,48 \cdot 6$ & $23 \cdot 9$ & $18 \cdot 2,29 \cdot 7$ & $51 \cdot 2$ & $49 \cdot 3,53 \cdot 0$ & $28 \cdot 0$ & $22 \cdot 8,33 \cdot 2$ & $47 \cdot 2$ & $45 \cdot 9,48 \cdot 5$ \\
\hline No & $49 \cdot 3$ & $48 \cdot 3,50 \cdot 4$ & $47 \cdot 0$ & $45 \cdot 0,48 \cdot 9$ & $51 \cdot 1$ & $50 \cdot 0,52 \cdot 0$ & $45 \cdot 3$ & $43 \cdot 5,47 \cdot 1$ & $50 \cdot 2$ & $49 \cdot 6,50 \cdot 8$ \\
\hline
\end{tabular}

WIC, Special Supplemental Nutrition Program for Women, Infants, and Children; PC, peer counselling.

*The total number of WIC participants who gave birth in the year 2006 was 29881 . However, 491 cases had missing values on the variable 'participant enrolment status', resulting in a combined total of 29390 for the prenatal cases and the postnatal cases. 
Table 4 Comparisons of breast-feeding initiation rates by participant enrolment status and agency PC status, Missouri, 2006

\begin{tabular}{|c|c|c|c|c|c|c|}
\hline & \multicolumn{2}{|c|}{ Breast-feeding initiation rate } & \multicolumn{2}{|c|}{ Crude model } & \multicolumn{2}{|c|}{ Adjusted* model } \\
\hline & $\%$ & $95 \% \mathrm{Cl}$ & OR & $95 \% \mathrm{Cl}$ & OR & $95 \% \mathrm{Cl}$ \\
\hline \multicolumn{7}{|l|}{ PC and enrolment status } \\
\hline Prenatal cases in non-PC agencies & $48 \cdot 8$ & $47 \cdot 8,49 \cdot 7$ & $1 \cdot 00$ & Reference & $1 \cdot 00$ & Reference \\
\hline Prenatal cases in PC agencies & $51 \cdot 1$ & $50 \cdot 1,51 \cdot 9$ & $1 \cdot 18$ & $1 \cdot 00,1 \cdot 40$ & $1 \cdot 21$ & $1 \cdot 03,1 \cdot 43$ \\
\hline Postnatal cases in non-PC agencies & $45 \cdot 2$ & $43 \cdot 4,47 \cdot 1$ & 0.85 & $0.78,0.93$ & $0 \cdot 81$ & $0.74,0.89$ \\
\hline Postnatal cases in PC agencies & $43 \cdot 8$ & $42 \cdot 1,45 \cdot 5$ & 0.88 & $0.73,1.05$ & $0 \cdot 86$ & $0 \cdot 72,1 \cdot 02$ \\
\hline \multicolumn{7}{|l|}{ Prenatal cases } \\
\hline \multicolumn{7}{|l|}{ Prenatal WIC enrolment duration } \\
\hline \multicolumn{7}{|l|}{ Non-PC agencies } \\
\hline$<3$ months & $42 \cdot 3$ & $40 \cdot 2,44 \cdot 4$ & $1 \cdot 00$ & Reference & $1 \cdot 00$ & Reference \\
\hline 3-6 months & $51 \cdot 8$ & $50 \cdot 1,53 \cdot 6$ & 1.51 & $1 \cdot 35,1 \cdot 69$ & $1 \cdot 56$ & $1 \cdot 39,1 \cdot 75$ \\
\hline$\geq 6$ months & $49 \cdot 5$ & $48 \cdot 1,50 \cdot 8$ & $1 \cdot 46$ & $1 \cdot 31,1 \cdot 62$ & $1 \cdot 51$ & $1 \cdot 35,1 \cdot 68$ \\
\hline \multicolumn{7}{|l|}{ PC agencies } \\
\hline$<3$ months & $43 \cdot 2$ & $41 \cdot 3,45 \cdot 2$ & $1 \cdot 17$ & $0.95,1.43$ & $1 \cdot 20$ & $0.99,1.47$ \\
\hline 3-6 months & $51 \cdot 4$ & $49 \cdot 5,52 \cdot 6$ & $1 \cdot 61$ & $1 \cdot 32,1 \cdot 97$ & $1 \cdot 71$ & $1 \cdot 42,2 \cdot 07$ \\
\hline$\geq 6$ months & $54 \cdot 5$ & $53 \cdot 2,55 \cdot 8$ & $1 \cdot 86$ & $1 \cdot 53,2 \cdot 26$ & $1 \cdot 95$ & $1 \cdot 61,2 \cdot 35$ \\
\hline \multicolumn{7}{|l|}{ PC agencies } \\
\hline \multicolumn{7}{|l|}{ Years of PC experience } \\
\hline $1-4$ & $46 \cdot 3$ & $44 \cdot 7,47 \cdot 9$ & $1 \cdot 00$ & Reference & $1 \cdot 00$ & Referencet \\
\hline $5-8$ & $53 \cdot 9$ & $52 \cdot 6,55 \cdot 3$ & $1 \cdot 51$ & $1 \cdot 22,1 \cdot 86$ & $1 \cdot 36$ & $1 \cdot 07,1 \cdot 72$ \\
\hline $9-14$ & $51 \cdot 9$ & $50 \cdot 3,53 \cdot 5$ & $1 \cdot 27$ & $1 \cdot 01,1 \cdot 59$ & $1 \cdot 06$ & $0 \cdot 85,1 \cdot 33$ \\
\hline \multicolumn{7}{|l|}{ Agency has IBCLC } \\
\hline Yes & $52 \cdot 0$ & $50 \cdot 3,53 \cdot 7$ & $1 \cdot 04$ & $0.81,1 \cdot 34$ & $1 \cdot 21$ & $1 \cdot 01,1 \cdot 45$ \\
\hline No & $50 \cdot 7$ & $49 \cdot 7,51 \cdot 8$ & $1 \cdot 00$ & Reference & $1 \cdot 00$ & Referencet \\
\hline \multicolumn{7}{|c|}{ Peer counsellor has other positions in WIC agency } \\
\hline Yes & $59 \cdot 0$ & $55 \cdot 7,62 \cdot 4$ & $1 \cdot 50$ & $1 \cdot 07,2 \cdot 12$ & $1 \cdot 01$ & $0 \cdot 72,1 \cdot 42$ \\
\hline No & $50 \cdot 5$ & $49 \cdot 6,51 \cdot 4$ & $1 \cdot 00$ & Reference & $1 \cdot 00$ & Referencet \\
\hline \multicolumn{7}{|c|}{$\begin{array}{l}\text { Breast-feeding coordinator is a Breastfeeding PC Task } \\
\text { Force member }\end{array}$} \\
\hline Force member & & & & & & \\
\hline Yes & $54 \cdot 0$ & $52 \cdot 3,55 \cdot 6$ & $1 \cdot 03$ & $0 \cdot 74,1 \cdot 45$ & 0.94 & $0 \cdot 68,1 \cdot 30$ \\
\hline No & $50 \cdot 0$ & $49 \cdot 0,51 \cdot 0$ & $1 \cdot 00$ & Reference & $1 \cdot 00$ & Referencet \\
\hline
\end{tabular}

PC, peer counselling; WIC, Special Supplemental Nutrition Program for Women, Infants, and Children; IBCLC, international board-certified lactation consultant.

*Adjusted for maternal age, race/ethnicity, education and family income unless specified otherwise.

†Adjusted for maternal age, race/ethnicity, education, family income and all other agency-level characteristics included in Table 1 (i.e. region, PC years, agency has IBCLC, peer counsellor has other positions the WIC agency, breast-feeding coordinator is a Breastfeeding PC Task Force member).

status and agency-level characteristics, the changes in the above results were negligible (Table 4).

\section{Effect of prenatal WIC enrolment duration on breast-feeding initiation among prenatal cases}

In both PC and non-PC agencies, women who were enrolled in WIC prior to the last three months antepartum were significantly more likely to initiate breast-feeding than those who were enrolled in WIC during the last three months antepartum. In PC agencies, the longer the duration of WIC enrolment, the higher was the breastfeeding initiation rate. While the initiation rates among women who were enrolled in WIC less than six months before delivery were similar between PC and non-PC agencies, the rate among women who were enrolled in WIC six months or more before delivery in PC agencies was 5 percentage points higher than that in non-PC agencies. There was significant interaction between prenatal WIC enrolment duration and agency PC status in multilevel analysis. After controlling for potential confounding factors, the effect of the PC programme on breast-feeding initiation was higher among women who were enrolled in WIC prior to the last six months antepartum (the ratio of odds ratios (ROR) between $\mathrm{PC}$ and non-PC agencies $=1 \cdot 95 / 1 \cdot 51=1 \cdot 29)$ than among those who were enrolled in WIC during the last six months antepartum $(\mathrm{ROR}=1 \cdot 20$ for those with $<3$ months of prenatal WIC enrolment; $\mathrm{ROR}=1 \cdot 71 / 1 \cdot 56=1 \cdot 10$ for those with 3-6 months of prenatal WIC enrolment; Table 4).

\section{Factors affecting breast-feeding initiation among prenatal cases in agencies with peer counselling}

Among PC agencies, those agencies having more years of breast-feeding PC experience, those with a peer counsellor who also had other positions in the agency, and those with a PC coordinator as a Breastfeeding PC Task Force member had significantly higher breast-feeding initiation rates. After adjusting for maternal sociodemographic characteristics and other agency-level characteristics, participants of PC agencies with 5-8 years of PC experience were more likely to initiate breastfeeding than those of PC agencies with less than 5 years of experience $(\mathrm{OR}=1.36$; $95 \%$ CI 1.07, 1.72). Similarly, participants of PC agencies with an IBCLC were more likely to initiate breast-feeding compared with participants of PC agencies without an IBCLC $(\mathrm{OR}=1 \cdot 21 ; 95 \%$ 
CI 1·01, 1·45; Table 4). However, the likelihood of breastfeeding initiation among participants of agencies with a peer counsellor who also had other positions in the agencies was not significantly different from that among participants of agencies with a peer counsellor who had no other positions in the agencies.

\section{Discussion}

Our study shows that prenatal participants of PC programmes are more likely to initiate breast-feeding compared with those with similar sociodemographic characteristics who participate in non-PC programmes. This finding is consistent with findings from several other studies conducted among WIC participants. Studies conducted among 108 Native American WIC participants in Utah found that PC increased the breast-feeding initiation rate $^{(10)}$. Among 242 African-American WIC participants in Baltimore, breast-feeding initiation was associated with attending 'peer counsellor only' intervention sites ${ }^{(11)}$. In a study conducted in rural West Tennessee, breast-feeding initiation in WIC participants was higher among women in the group with a peer counsellor $(n 156)$ than among those without such a counsellor $(n 135)^{(12)}$. The PC programme increased breast-feeding initiation and duration among seventy-two rural low-income women in Iowa ${ }^{(13)}$. A randomized trial conducted in a predominantly Latina low-income community found that PC significantly increased the breast-feeding initiation rate ${ }^{(14)}$.

While most of the aforementioned studies involved small study sizes, one study used the Mississippi statewide PedNSS data for 1989 and $1993^{(15)}$. That study found that breast-feeding initiation rose significantly more sharply in WIC clinics with PC programmes than in those without PC programmes ${ }^{(15)}$. Besides a large sample size, a major strength of the Mississippi study was that it compared the changes between PC and non-PC agencies in breast-feeding initiation before and after the PC programmes were implemented. However, that study included only two data points in the analysis - one year before and one year after the implementation of the PC programme; while in any given state, breast-feeding initiation rates are known to fluctuate from year to year. Also, the Mississippi study failed to describe and control for potential confounding factors.

As with the Mississippi study, our study also had a large study size and involved a state-wide system. There are, however, significant differences between the two studies. First, the operations of the PC programmes are different. The staff in the Mississippi PC programme worked an average of $10 \mathrm{~h} /$ week and contacted forty to forty-nine different participants each month ${ }^{(15)}$, whereas peer counsellors in the Missouri PC programme worked approximately the same number of hours per week and served a median of 216 different participants each month in 2006. Additionally, unlike the Mississippi PC programme, not all WIC participants in Missouri PC agencies were contacted by peer counsellors. Also, our study controlled for multiple potential confounding factors. The fact that both studies have found similar results despite differences in study designs suggests that PC improves breast-feeding initiation not only in clinical trial settings ${ }^{(6-8,14)}$ but in public health programme settings as well.

In addition to finding a positive impact of the PC programme on breast-feeding initiation, our study also shows that the impact is highest among those who enrol in WIC for a longer duration before delivery. Therefore, to maximize the benefit of a PC programme, measures should be taken to enrol eligible prenatal women into WIC early. Our study also shows that compared with prenatal cases, postpartum cases of similar sociodemographic characteristics are less likely to initiate breast-feeding in both PC and non-PC agencies. This could be an indication that prenatal participation of WIC itself increases breast-feeding initiation. However, because of self-selection, postpartum cases may not be representative of those who are eligible but did not participate in WIC prenatally.

A major limitation of our study was that PC agencies were self-selected to participate in the programmes. These agencies might have certain unmeasured attributes that were potentially associated with increased breastfeeding initiation, but the analysis had not completely controlled for these attributes.

On the other hand, our study had at least three strengths. First, it included all 2006 Missouri WIC participants who gave birth in 2006. The large study size increased the power of the study and allowed for the control of a large number of sociodemographic, behavioural and other characteristics of the mothers, newborns and WIC agencies. Second, by including postpartum cases in the analysis, we found similar lower levels of breast-feeding initiation among postnatal cases between PC and non-PC agencies, further strengthening our study since PC is unlikely to increase breast-feeding initiation for postpartum cases. Third, the present study used the multilevel analytical technique, which properly accounted for the variance structures in the database.

\section{Conclusions}

Our study shows that prenatal participants of WIC PC agencies are more likely to initiate breast-feeding compared with prenatal participants of non-PC agencies in Missouri, and the increase is highest among participants who enrol in WIC prior to the last six months antepartum. Compared with prenatal cases, postpartum cases of both $\mathrm{PC}$ and non-PC agencies are less likely to initiate breastfeeding. PC agencies with 5-8 years of PC experience have a higher rate of breast-feeding initiation than agencies 
with 1-4 years of experience. PC agencies with an IBCLC also have a higher rate of breast-feeding initiation than PC agencies without an IBCLC.

Our study, along with the Mississippi study and other studies conducted in different settings, shows that PC programmes are effective in improving breast-feeding initiation rates ${ }^{(10-15)}$. Our study also suggests that nutrition programmes focusing on breast-feeding should enrol participants early in the prenatal period and have an IBCLC.

\section{Acknowledgements}

The study was supported with funding from the USDA Missouri WIC Program (grant 3MO700704). Analysis was supported with data from the USDA Missouri WIC Breastfeeding Peer Counseling Program (grant 3MO700804). There is no conflict of interests related to this study. S.Y. contributed to conceptualization and design of the study, analysis of data and preparation of the article. Q.L. contributed to management and analysis of data. K.M., C.K., M.W. and P.F. contributed to data collection and preparation of the article. B.-P.Z. contributed to study design and preparation of the article. The authors would like to thank $\mathrm{Mr}$ Brian Tordoff for proofreading.

\section{References}

1. Turck D (2005) Breastfeeding health benefit for child and mother. Arch Pediatr 2, Suppl. 3, S145-S165.

2. Collaborative Group on Hormonal Factors in Breast Cancer (2002) Breast cancer and breastfeeding: collaborative reanalysis of individual data from 47 epidemiological studies in 30 countries, including 50302 women with breast cancer and 96973 women without the disease. Lancet 360, 187-195.

3. Leung AK \& Sauve RS (2005) Breast is best for babies. J Natl Med Assoc 97, 1010-1019.
4. Oddy WH (2002) The impact of breast milk on infant and child health. Breastfeed Rev 10, 5-18.

5. Walker WA (2005) The dynamic effects of breastfeeding on intestinal development and host defense. Adv Exp Med Biol 554, 155-170.

6. Dennis CL, Hodnett E, Gallop R \& Chalmers B (2002) The effect of peer support on breast-feeding duration among primiparous women: a randomized controlled trial. CMAJ 166, 21-28.

7. Chapman DJ, Damio G, Young S, Chapman DJ \& PérezEscamilla R (2004) Effectiveness of breastfeeding peer counseling in a low-income, predominantly Latina population: a randomized controlled trial. Arch Pediatr Adolesc Med 158, 897-902.

8. Merewood A, Chamberlain LB, Cook JT, Philipp BL, Malone K \& Bauchner H (2006) The effect of peer counselors on breastfeeding rates in the neonatal intensive care unit: results of a randomized controlled trial. Arch Pediatr Adolesc Med 160, 681-685.

9. United States Department of Agriculture (2007) WIC Learning Center. http://www.nal.usda.gov/wicworks/ Learning_Center/support_peer.html (accessed August 2007).

10. Long DG, Funk-Archuleta MA, Geiger CJ, Mozar AJ \& Hines JN (1995) Peer counselor program increases breastfeeding rates in Utah Native American WIC population. J Hum Lact 11, 279-284.

11. Caulfield LE, Gross SM, Bentley ME, Bronner Y, Kessler L, Jensen J, Weathers B \& Paige DM (1998) WIC-based interventions to promote breastfeeding among AfricanAmerican women in Baltimore: effects on breastfeeding initiation and continuation. J Hum Lact 14, 15-22.

12. Shaw E \& Kaczorowski J (1999) The effect of a peer counseling program on breastfeeding initiation and longevity in a low-income rural population. J Hum Lact 15, 19-25.

13. Schafer E, Vogel MK, Viegas S \& Hausafus C (1998) Volunteer peer counselors increase breastfeeding duration among rural low-income women. Birth 25, 101-106.

14. Anderson AK, Damio G, Young S, Chapman DJ \& PérezEscamilla R (2005) A randomized trial assessing the efficacy of peer counseling on exclusive breastfeeding in a predominantly Latina low-income community. Arch Pediatr Adolesc Med 159, 836-841.

15. Grummer-Strawn LM, Rice SP, Dugas K, Clark LD \& Benton-Davis S (1997) An evaluation of breastfeeding promotion through peer counseling in Mississippi WIC clinics. Matern Child Health J 1, 35-42. 